\title{
Significant contribution of HONO to secondary pollutants during a severe winter pollution event in southern China
}

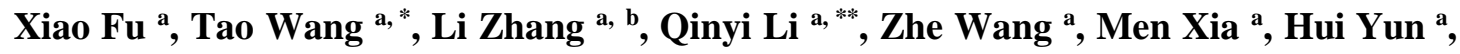
Weihao Wang a, Chuan Yu a, Dingli Yue c, Yan Zhou ${ }^{c}$, Junyun Zheng d, Rui Han ${ }^{\text {e }}$

${ }^{a}$ Department of Civil and Environmental Engineering, Hong Kong Polytechnic University, Hong Kong 99907, China

${ }^{\mathrm{b}}$ Atmospheric and Oceanic Sciences, Princeton University, NOAA, Princeton, New Jersey 08540, United States

${ }^{\mathrm{c}}$ Guangdong Provincial Environmental Monitoring Center, Guangzhou, China

${ }^{\mathrm{d}}$ Institute for Environmental and Climate Research, Jinan University, Guangzhou, China

${ }^{\mathrm{e}}$ National Meteorological Information Center, China Meteorological Administration, Beijing 100081, China

* Correspondence to: Tao Wang (tao.wang@polyu.edu.hk)

** Now at: Department of Atmospheric Chemistry and Climate, Institute of Physical Chemistry Rocasolano, CSIC, Madrid 28006, Spain 
Table S1. Performance statistics of meteorological variables for the innermost domain

\begin{tabular}{|c|c|c|c|c|}
\hline Variable & $\mathrm{T} 2{ }^{\mathrm{c}}\left({ }^{\circ} \mathrm{C}\right)$ & $\mathrm{H} 2^{\mathrm{c}}(\mathrm{g} / \mathrm{kg})$ & $\mathrm{WS} 10^{\mathrm{c}}\left(\mathrm{m} \mathrm{s}^{-1}\right)$ & $\mathrm{WD} 10^{\mathrm{c}}\left(^{\circ}\right)$ \\
\hline Mean OBS ${ }^{a, b}$ & 20.8 & 13.3 & 2.4 & 229.2 \\
\hline Mean SIM ${ }^{\mathrm{b}}$ & 20.4 & 12.2 & 2.1 & 232.5 \\
\hline $\mathrm{MB}^{\mathrm{b}}$ & -0.4 & -1.1 & -0.2 & 3.3 \\
\hline $\operatorname{NMB}^{\mathrm{b}}(\%)$ & -2.0 & -8.1 & -8.9 & 1.4 \\
\hline $\operatorname{NME}^{\mathrm{b}}(\%)$ & 5.1 & 11.2 & 41.7 & 24.6 \\
\hline
\end{tabular}

a The observation data was obtained from the National Climatic Data Center (NCDC) (https://www7.ncdc.noaa.gov/CDO/cdo ).

${ }^{\text {b }}$ Mean OBS: average observed values; Mean SIM: average simulated values; MB: bias; NMB: normalized mean bias; NME: normalized mean error.

${ }^{\mathrm{c}} \mathrm{T} 2$ : temperature at 2 meter; $\mathrm{H} 2$ : specific humidity at 2 meter; WS10: wind speed at 10 meter; WD10: wind direction at 10 meter.
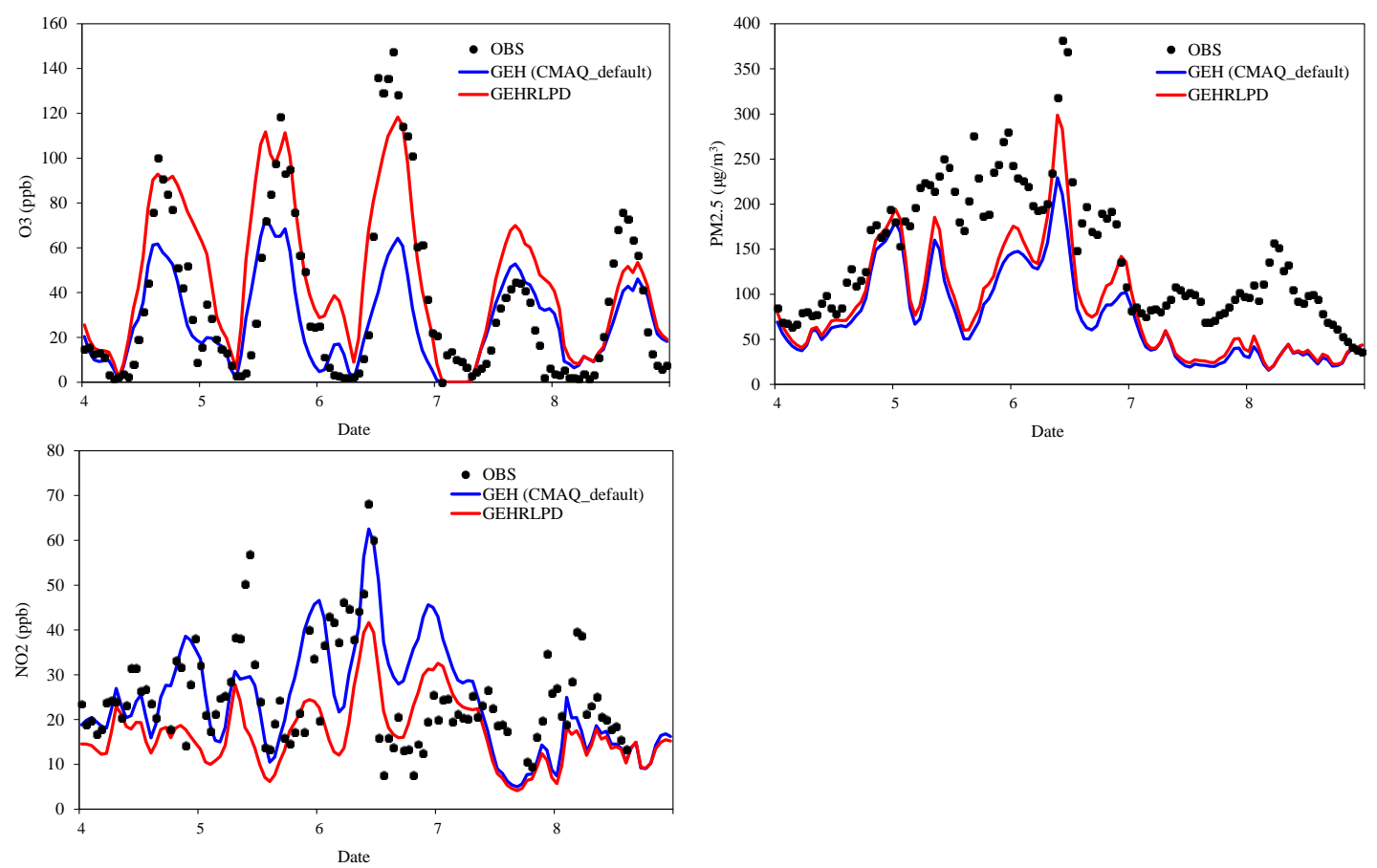

Fig. S1. Observed and simulated $\mathrm{O}_{3}, \mathrm{PM}_{2.5}$ and $\mathrm{NO}_{2}$ concentrations by the default and revised CMAQ model at Heshan site 

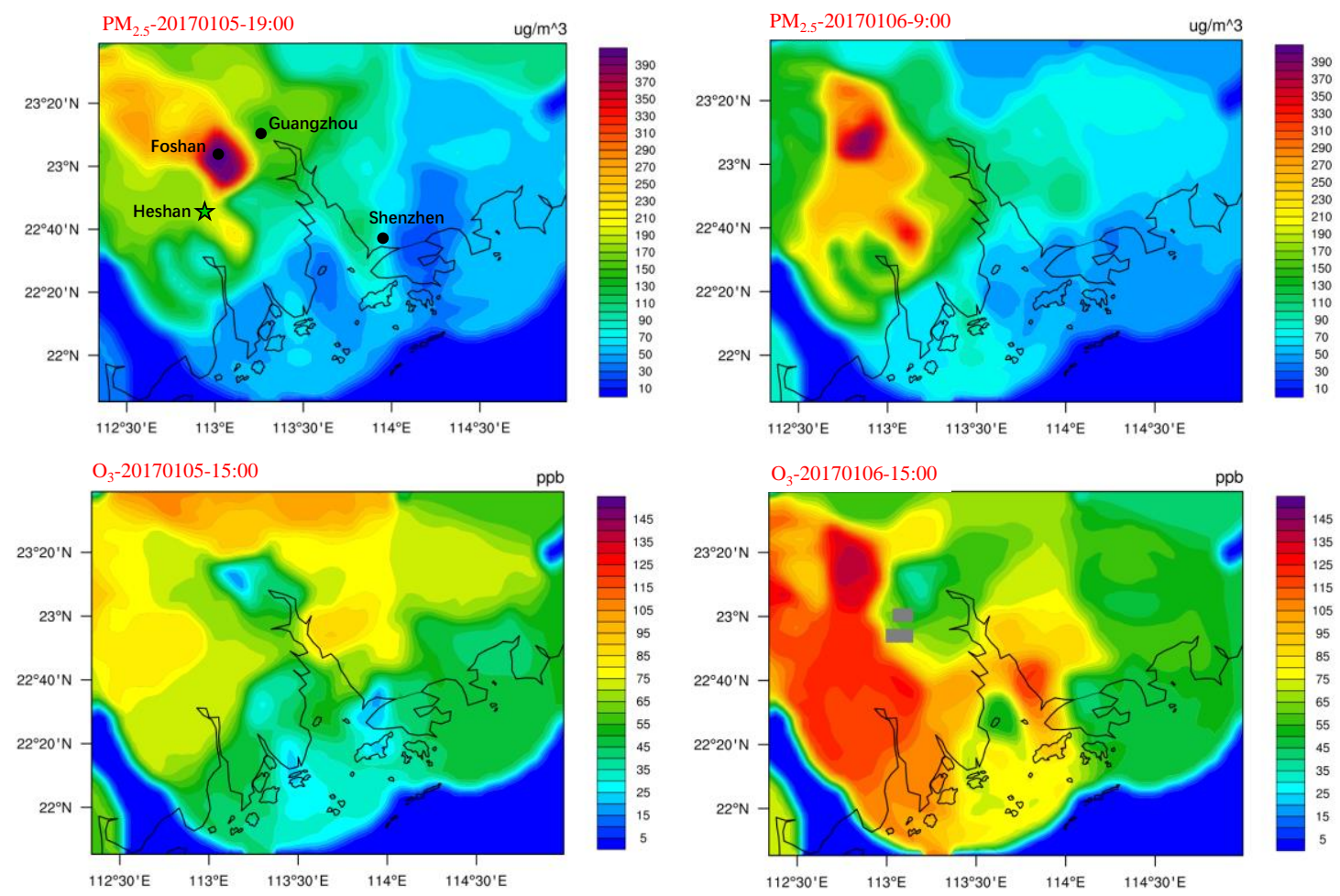

Fig. S2. Spatial distribution of observed $\mathrm{PM}_{2.5}$ (19:00 on 5 January and 9:00 on 6 January) and $\mathrm{O}_{3}(15: 00$ on 5 and 6 January) concentrations
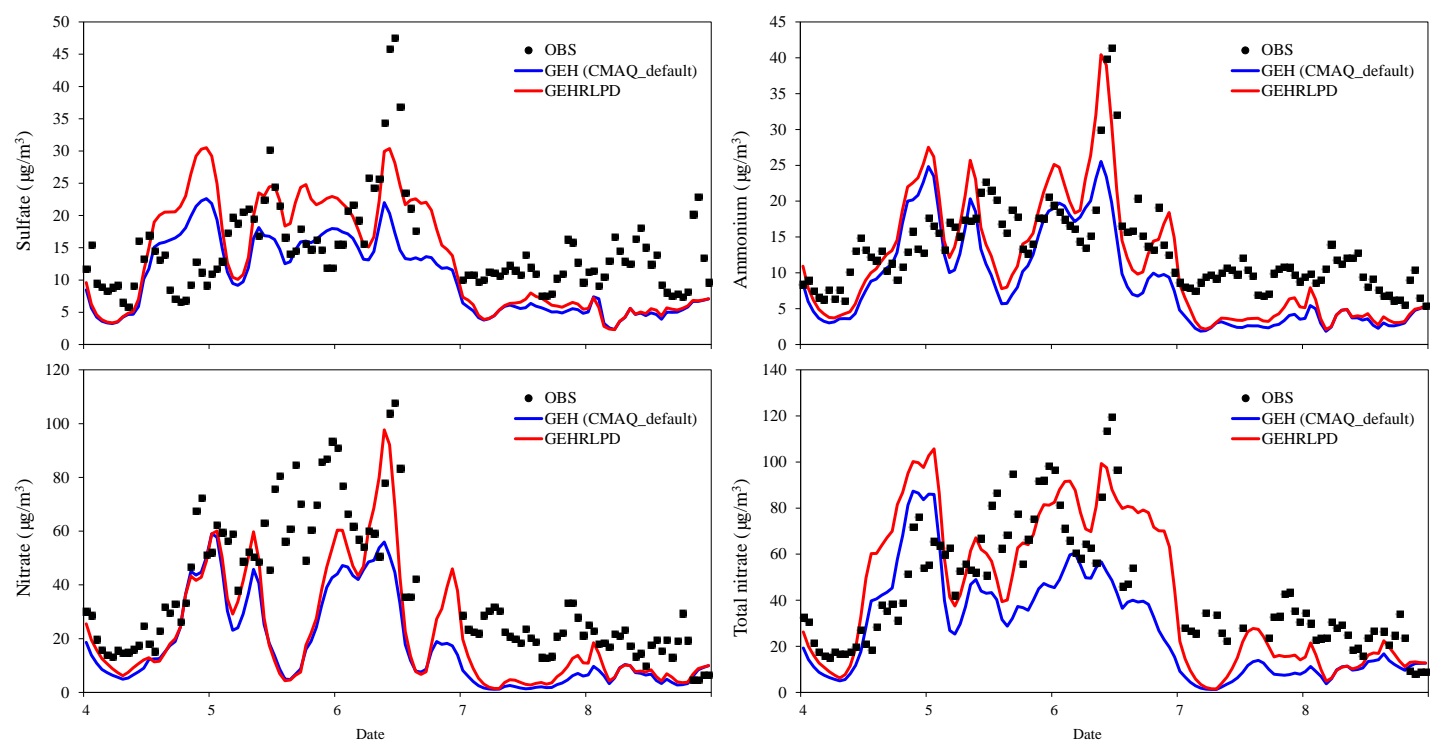

Fig. S3. Observed and simulated particle sulfate, ammonium, nitrate, and total nitrate concentrations by the default and revised CMAQ model at Heshan site 

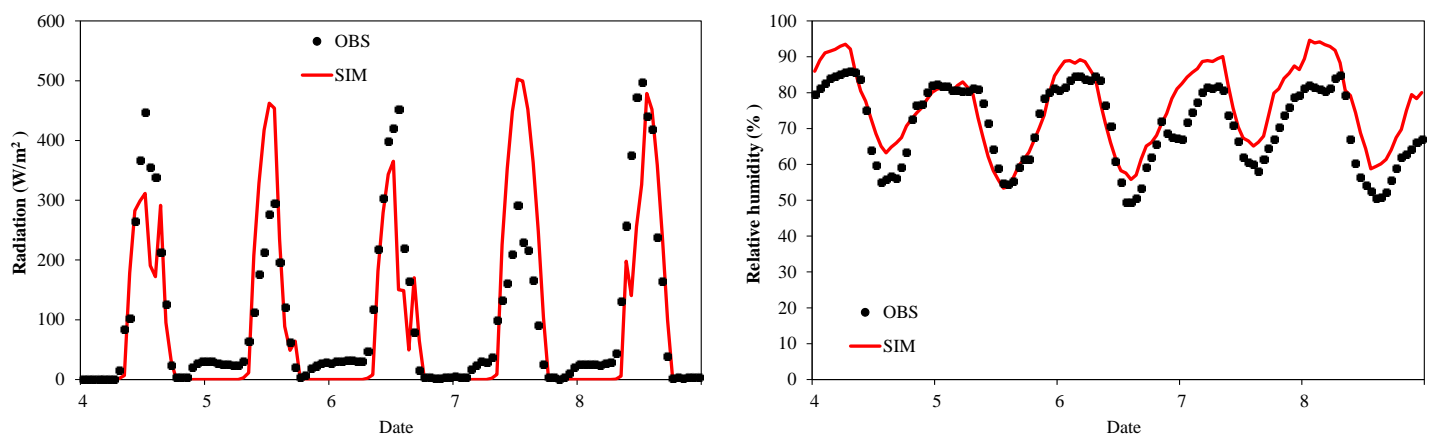

Fig. S4. Diurnal variations of observed and simulated radiation and relative humidity at Heshan site

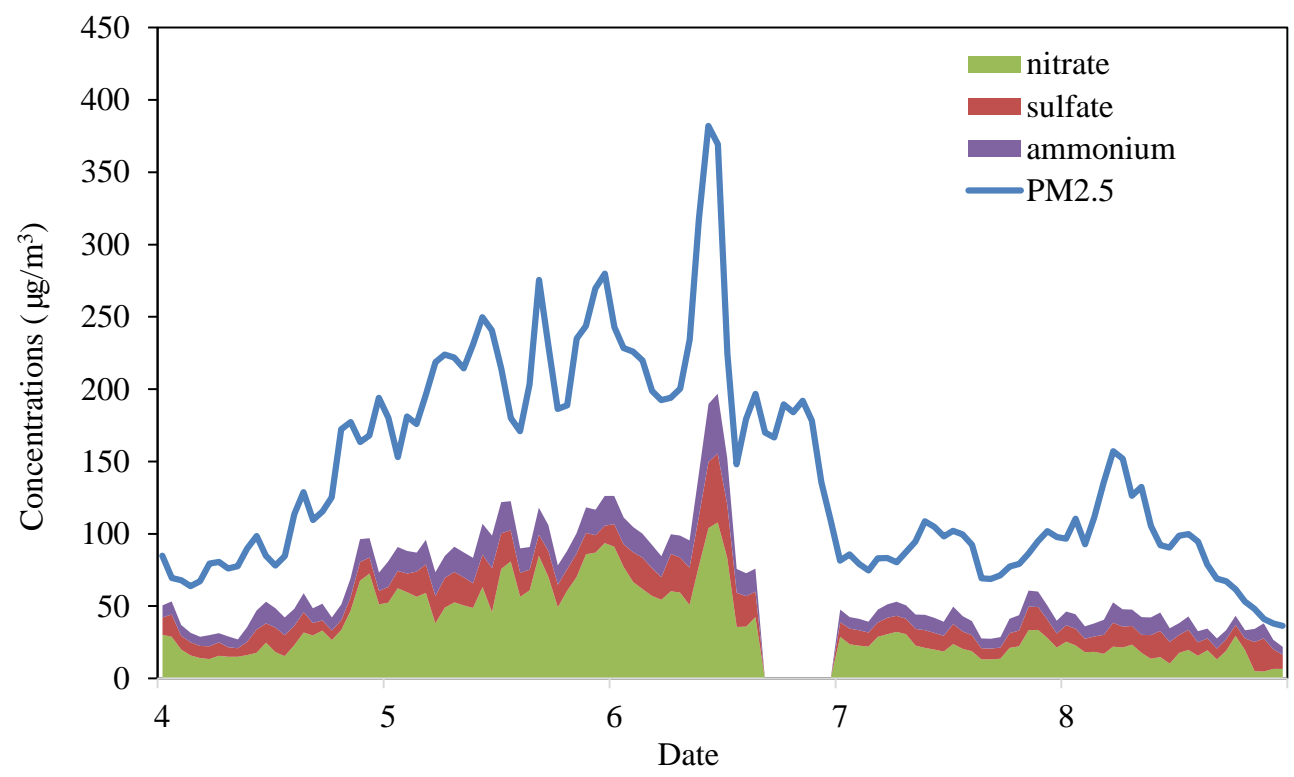

Fig. S5. Observed hourly variations of nitrate, sulfate, ammonium and $\mathrm{PM}_{2.5}$ concentrations during 4-8 January, 2017 at Heshan site. 